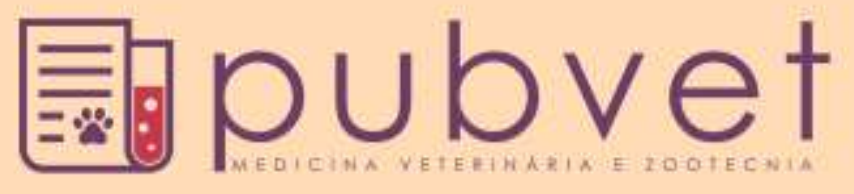

https://doi.org/10.22256/pubvet.v12n1a5.1-7

\title{
Vertebrados silvestres atropelados na rodovia BR-230, Paraíba, Brasil
}

\author{
Marcela Meira Ramos-Abrantes ${ }^{\left(1,2^{*}\right.}$, Artur da Nóbrega Carreiro ${ }^{\circledR}$, Débora Vitória \\ Fernandes de Araújo ${ }^{6}$, Joyce Galvão de Souza ${ }^{6}$, João Paulo Rodrigues de Lima ${ }^{6}$, \\ Hyldetan Ruan de Araújo Cezar ${ }^{65}$, Lucas Silva Leite ${ }^{6}$, Stephenson Hallison Formiga \\ Abrantes 2,6
}

${ }^{1}$ Faculdades Integradas de Patos, Centro Educacional de Ensino Superior de Patos, Patos-PB, Brasil.

${ }^{2}$ Programa de Pós-Graduação em Ciência Animal Tropical, Universidade Federal Rural de Pernambuco, Brasil.

${ }^{3}$ Programa de Pós-Graduação em Medicina Veterinária, Universidade Federal de Campina Grande, Campus Patos. E-mail:

${ }^{4}$ Universidade Federal de Campina Grande, Unidade Acadêmica de Medicina Veterinária, Patos, Paraíba, Brasil. E-mail:

${ }^{5}$ Unidade Acadêmica de Ciências Biológicas, Universidade Federal de Campina Grande, Patos, Paraíba, Brasil. E-mail:

${ }^{6}$ Universidade Federal de Campina Grande, Unidade Acadêmica de Ciências Biológicas. E-mail: stephensonabrantes@yahoo.com.br Autor para correspondência, E-mail: marcelabio@gmail.com

RESUMO. Os atropelamentos de animais silvestres têm recebido especial atenção dos pesquisadores nos últimos anos por se tratar da causa primária de morte em estradas. Contudo, estudos que abordem o tema ainda são escassos e, geralmente, tratam apenas de mamíferos. Diante disso, este estudo teve o objetivo de monitorar a mortalidade de animais silvestres por atropelamentos no trecho Campina Grande - Patos da BR-230 entre novembro de 2010 e novembro de 2011. Foram realizadas viagens mensais com duração média de $2 \mathrm{~h}$, percorrendo-se o trecho de carro. Foi comparado o número de atropelamentos no período seco e chuvoso, geral e por grupo. Os animais atropelados foram identificados até o menor nível taxonômico possível, descartando-se os animais domésticos. Foram registrados 188 espécimes de vertebrados atropelados, sendo Mammalia o mais amostrado $(\mathrm{n}=108 ; 57,4 \%)$. A espécie mais representativa foi Cerdocyon thous $(\mathrm{n}=87 ; 46,5 \%)$. O maior número de atropelamentos ocorreu no período seco. Quando comparado por grupo, porém, aves e répteis apresentaram um maior número de atropelamentos durante o período chuvoso. Cerdocyon thous, apresentou taxas de atropelamento maiores que em outros levantamentos realizados no Brasil ( 87 indivíduos, 46,5\%), o que parece ser uma realidade local. Dentre os répteis atropelados, $70 \%$ corresponderam a espécies de serpentes, que pode estar relacionado ao deslocamento lento e necessidade de termorregulação desses animais e pelo fato de serem considerados "animais perigosos", levando ao extermínio intencional.

Palavras chave: conservação, ecologia de estradas, caatinga

\section{Wild vertebrate's roadkill on the BR-230 Highway, State of Paraíba, Brazil}

ABSTRACT. The trampling of wild animals have received special attention from researchers in recent years because it is the primary cause of death on roads. However, studies that address the topic are still scarce and generally deal only with mammals. Therefore, this study aimed to monitor the mortality of wild animals by trampling between Campina Grande and Patos in the BR-230 highway from November 2010 to November 2011. Were made monthly trips lasting 2 hours traveled by car. We compared the number of trampling in the dry and rainy period. The animals killed on roads were identified to lowest possible taxonomic level, discarding pets. We recorded 188 specimens of vertebrates' trampling, and the most sampled were mammals $(n=108,57.4 \%)$. The most 
representative species was Cerdocyon thous ( $\mathrm{n}=87,46.5 \%)$. The highest number of trampling occurred in the dry season. When compared by group, however, birds and reptiles had a greater number of trampling during the rainy season. Cerdocyon thous presented trampling rates higher than in other surveys conducted in Brazil (87 specimens, 46.5\%), which seems to be a local reality. Among the reptiles get hit, $70 \%$ corresponded to species of snakes, which can be related to the slow movement and need for thermoregulation of these animals and because they are considered "dangerous animals", leading to intentional killing.

Keywords: conservation, road ecology, Caatinga

\section{Vertebrados silvestres atropellados en la carretera BR-230, Paraíba, Brasil}

RESUMEN. Los atropellamientos de animales silvestres han recibido especial atención de los investigadores en los últimos años por tratarse de la causa primaria de muerte en carreteras. Sin embargo, estudios que abordan el tema todavía son escasos y, generalmente, tratan sólo de mamíferos. Frente a eso, este estudio tuvo el objetivo de monitorear la mortalidad de animales silvestres por atropellamientos en el tramo Campina Grande - Patos de la BR-230 entre noviembre de 2010 y noviembre de 2011. Se realizaron viajes automovilísticos mensualmente con duración promedio de $2 \mathrm{~h}$. Se comparó el número de atropellamientos en el período seco y lluvioso, general y por grupo. Los animales atropellados fueron identificados hasta el menor nivel taxonómico posible, descartándose los animales domésticos. Se registraron 188 especímenes de vertebrados atropellados, siendo Mammalia el más muestreado $(\mathrm{n}=108 ; 57,4 \%)$. La especie más representativa fue Cerdocyon thous $(\mathrm{n}=87,46,5 \%)$. El mayor número de atropellos ocurrió en el período seco. Sin embargo, al compararlos por grupo, las aves y los reptiles presentaron un mayor número de atropellamientos durante el período lluvioso. Cerdocyon thous, presentó tasas de atropellamiento mayores que en otros levantamientos realizados en Brasil (87 individuos, 46,5\%), lo que parece ser una realidad local. Entre los reptiles atropellados, el $70 \%$ correspondía a especies de serpientes, que puede estar relacionado al desplazamiento lento y necesidad de termorregulación de esos animales y por el hecho de ser considerados "animales peligrosos", llevando al exterminio intencional.

Palabras clave: conservación, ecología de carreteras, Caatinga

\section{Introdução}

A construção de estradas é uma importante causa de desequilíbrio ecológico, podendo ocasionar diversos impactos, como: alterações no comportamento dos animais; alteração do estado fisiológico; introdução de espécies exóticas devido a alterações do hábitat; estresse e/ou remoção de espécies nativas; modificação de cadeia alimentar; fragmentação e alteração de habitats por efeito de borda; interceptação dos corredores de dispersão natural da fauna terrestre; isolamento populacional e perda de indivíduos por colisão com veículos (Trombulak \& Frissell, 2000).

Além desses impactos provocados pela construção de rodovias, pode-se listar o atropelamento de animais silvestres, causa primária de morte em estradas (Cherem et al., 2007). A presença da rodovia na área de vida de determinadas espécies e a disponibilidade de alimento ao longo das rodovias são as principais causas dos atropelamentos (Lima \& Obara, 2004). Segundo Sousa \& Miranda (2010), a mortalidade por atropelamento pode ser altamente impactante para populações naturais, principalmente para as espécies ameaçadas de extinção e as que possuem áreas de vida relativamente grandes e taxas reprodutivas baixas.

Nos últimos anos tem aumentado o número de animais atropelados em estradas e rodovias, especialmente vertebrados, em todo mundo (Trombulak \& Frissell, 2000), o que tem despertado o interesse de pesquisadores. Contudo, no Brasil ainda são escassos os estudos que abordam esse tema (Turci \& Bernarde, 2009). Além de escassos, alguns deles só abordaram mamíferos (Vieira, 1996, Pereira et al., 2006, Cherem et al., 2007). Assim, faz-se necessária a 
realização de trabalhos que visem monitorar os atropelamentos de vários grupos de animais silvestres nas rodovias brasileiras.

Diante disso, este trabalho teve o objetivo de monitorar a mortalidade de animais silvestres por atropelamentos no trecho Campina Grande - Patos da BR-230.

\section{Material e Métodos}

O estudo foi realizado na Rodovia Federal BR230. Esta rodovia tem a extensão de 4.965 quilômetros, ligando as cidades de Cabedelo (PB) e Benjamin Constant (AM), cruzando o Brasil no sentido leste-oeste (DNIT, 2011). O trecho monitorado tem extensão de 180 quilômetros e liga os municípios de Campina Grande e Patos, no estado da Paraíba, Brasil (Figura 1). O trecho encontra-se em uma região marcada por uma forte sazonalidade no regime de chuvas, não só na variação dos totais pluviométricos, mas, principalmente, da duração e intensidade dessas precipitações (Silva et al., 2009). A vegetação da área estudada é constituída de uma transição entre agreste, nas áreas próximas a cidade de Campina Grande (Moreira, 1989) e Caatinga nas áreas próximas a cidade de Patos (Velloso et al., 2002).

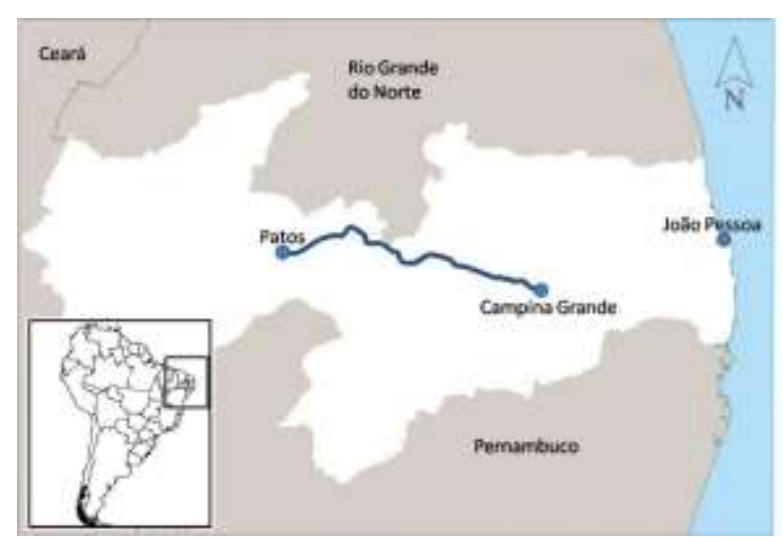

Figura 1. Localização do Estado da Paraíba, destacando o trecho da Rodovia BR-230 monitorado.

\section{Procedimentos Metodológicos}

Os espécimes atropelados foram registrados em viagens mensais, 12 ao todo, realizadas entre novembro de 2010 e outubro de 2011. A cada viagem foram percorridos $180 \mathrm{~km}$, totalizando $2340 \mathrm{~km}$ ao final do monitoramento. As amostragens foram realizadas no período diurno, sempre por dois coletores, seguindo uma velocidade de $80 \mathrm{Km} / \mathrm{h}$, parando-se o carro a cada avistamento, para confirmação. Não foram registrados os atropelamentos de animais domésticos. Foram realizadas sete viagens no período seco e seis no período chuvoso. Os espécimes atropelados foram identificados até o menor nível taxonômico possível. Foi calculada a taxa média de vertebrados atropelados utilizando a relação entre o número de indivíduos registrados e o total de quilômetros percorridos (Turci \& Bernarde, 2009). Para verificar se houve diferença no número de indivíduos atropelados nas diferentes estações (chuva e seca) foi aplicado teste não paramétrico Qui-quadrado com 5\% de significância, utilizando o programa Bioestat 5.0 (Ayres, 2007).

\section{Resultados}

Foram registrados 188 espécimes de vertebrados atropelados (Tabela 1). Mammalia foi o grupo mais representativo com 108 espécimes $(57,4 \%)$ registrados, seguido por Reptilia com 37 exemplares (19,7\%), Aves $(14,3 \%)$ e Amphibia $(8,6 \%)$. Cerdocyon thous (Linnaeus, 1766) foi a espécie com maior número de indivíduos atropelados, sozinha correspondeu a quase metade dos registros $(\mathrm{N}=87 ; 46,5 \%)$ (Tabela 1$)$.

A curva do coletor não atingiu a assíntota (Figura 2), entretanto no nono mês de amostragem $96,4 \%$ das espécies já tinham sido registradas. Foi encontrada uma diferença significativa no número total de indivíduos atropelados entre os períodos seco e chuvoso $\left(X^{2}=23,94 ; \mathrm{gl}=3 ; \mathrm{p}=0.0001\right)$, sendo o maior número de registros verificados durante a estação seca (Tabela 1 e Figura 3), quando comparado por grupo, aves e répteis apresentaram um maior número de indivíduos atropelados registrados durante a estação chuvosa (Tabela 1).

\section{Discussão}

Apesar de detectado um alto número de atropelamentos ao longo deste estudo, este valor pode estar subestimado, uma vez que a curva de suficiência amostral não atingiu a assintota, evidenciando a nessecidade de um período maior de amostragem. Além disso, os animais podem ser atropelados e vir a morrer em outros locais que não foram amostrados, como levantado por Turci \& Bernarde (2009) ou serem removidos por mamíferos carnívoros e aves de rapina que se alimentam de carcaças de outros animais.

Dados apontam para o fato de que animais são atropelados não só por estarem próximos a uma área de rodovia. Alguns podem ser atraídos por restos de alimentos e lixo descartados nas estradas 
pelos motoristas (Scoss et al., 2004, Sousa \& Miranda, 2010). Espécies necrófagas são atraídas pelas carcaças dos animais mortos nas estradas e à noite espécies de grandes predadores se utilizam de rodovias com tráfico reduzido para se locomoverem, perpetuando assim um ciclo de atropelamentos (Scoss et al., 2004).

Tabela 1. Relação dos vertebrados atropelados registrados na Rodovia BR - 230 na Paraíba entre os municípios de Campina Grande e Patos entre Novembro de 2010 e Outubro de 2011. ( $\mathrm{N}$ = número de indivíduos).

\begin{tabular}{|c|c|c|c|}
\hline \multirow{2}{*}{ Táxon } & \multirow{2}{*}{$\mathbf{N}(\%)$} & \multicolumn{2}{|c|}{ Estação } \\
\hline & & Seca, $\mathbf{n}$ & Chuvosa, $n$ \\
\hline Amphibia & $16(8,6 \%)$ & 12 & 4 \\
\hline Rhinela $\mathrm{sp}$ & $16(8,6 \%)$ & 12 & 4 \\
\hline Reptilia & $37(19,7 \%)$ & 17 & 20 \\
\hline Amphisbaena $\mathrm{sp}$ & $3(1,6 \%)$ & 2 & 1 \\
\hline Ameiva ameiva (Linnaeus, 1758) & $2(1.1 \%)$ & 1 & 1 \\
\hline Tupinambis merianae (Duméril \& Bibron, 1839). & $1(0,5 \%)$ & - & 1 \\
\hline Iguana iguana (Linnaeus, 1758) & $4(2,1 \%)$ & 2 & 2 \\
\hline Boa constrictor (Linnaeus, 1758) & $5(2,7 \%)$ & 3 & 2 \\
\hline Epicrates cenchria (Linnaeus,1758) & $2(1,1 \%)$ & 1 & 1 \\
\hline Boiruna sertaneja (Zaher 1996) & $3(1,6 \%)$ & - & 3 \\
\hline Philodryas nattereri (Steindachner, 1870) & $9(4,8 \%)$ & 4 & 5 \\
\hline Pseudoboa nigra (Duméril, Bribon \& Duméril, 1854) & $6(3,2 \%)$ & 3 & 3 \\
\hline Philodryas olfersii (Lichtenstein, 1823) & $1(0,5 \%)$ & 1 & - \\
\hline Phrynops sp. (Wagler, 1830) & $1(0,5 \%)$ & - & 1 \\
\hline Aves & $27(14,3 \%)$ & 8 & 19 \\
\hline Crotophaga $\mathrm{sp}$ & $1(0,5 \%)$ & - & 1 \\
\hline Caprimulgus sp & $2(1,1 \%)$ & 1 & 1 \\
\hline Pitangus sp & $1(0,5 \%)$ & - & 1 \\
\hline Polyborus plancus (Miller, J. F., 1777) & $5(2,7 \%)$ & 2 & 3 \\
\hline Coragyps sp. & $2(1,1 \%)$ & - & 2 \\
\hline Passeriformes não identificados & $16(8,5 \%)$ & 5 & 11 \\
\hline Mammalia & $108(57,4 \%)$ & 80 & 28 \\
\hline Cerdocyon thous (Linnaeus, 1766) & $87(46,5 \%)$ & 66 & 21 \\
\hline Puma yagouaroundi (Geoffroy, 1803) & $1(0,5 \%)$ & 1 & - \\
\hline Conepatus semistriatus(Boddaert, 1785) & $4(2,1 \%)$ & 3 & 1 \\
\hline Galictis sp & $5(2,7 \%)$ & 3 & 2 \\
\hline Procyon cancrivorus (G. Cuvier, 1798) & $1(0,5 \%)$ & 1 & - \\
\hline Didelphis albiventris (Lund 1840) & $4(2,1 \%)$ & 1 & 3 \\
\hline Kerodon rupestris (Wied-Neuwied, 1820) & $3(1,6 \%)$ & 2 & 1 \\
\hline Tamandua tetradactyla (Linnaeus, 1758) & $1(0,5 \%)$ & 1 & - \\
\hline Não identificados & $2(1,1 \%)$ & 2 & - \\
\hline Total & $188(100 \%)$ & 117 & 71 \\
\hline
\end{tabular}

Os mamíferos foram os animais mais registrados durante o período analisado. Muitos trabalhos citam a grande mortalidade de cachorro do mato, Cerdocyon thous, por colisão com 
automóveis (Gumier \& Sperber, 2009, Turci \& Bernarde, 2009, Sousa \& Miranda, 2010, Sobanski et al., 2012), corroborando com os resultados encontrados no presente estudo. Esta parece ser uma realidade local, uma vez que taxa semelhante foi obtida em outro trecho da mesma rodovia por Sousa \& Miranda (2010). Este fato pode ser explicado pelos cachorros-do-mato serem predadores e carniceiros e se moverem intensamente ao longo de estradas, procurando presas ou outros animais mortos por atropelamento. Além disto, trata-se de um animal que apresenta grande agilidade e uma ampla área de vida (Beisiegel, 1999, Sousa \& Miranda, 2010), possibilitando seu deslocamento por grandes distâncias a procura de alimento. A vegetação as margens da estrada, ou as próprias faixas de rolagem, podem ser utilizadas por estes animais como trilhas artificiais para sua movimentação, aumentando assim as chances de serem atropeladas (Sousa \& Miranda, 2010).

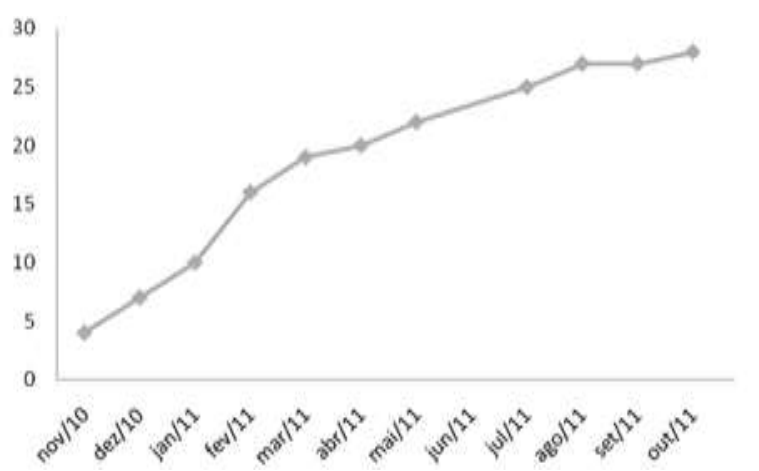

Figura 2. Curva do coletor das espécies durante o período de amostragem entre Novembro de 2010 e Outubro de 2011.

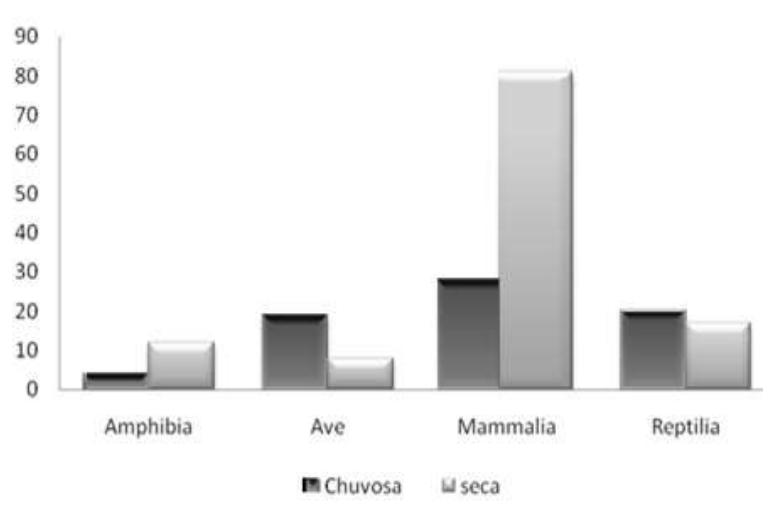

Figura 3. Vertebrados atropelados durante a estação chuvosa e seca, BR 230, Paraíba, Brasil.

Os mamíferos foram os animais mais registrados durante o período analisado. Muitos trabalhos citam a grande mortalidade de cachorro do mato, Cerdocyon thous, por colisão com automóveis (Gumier \& Sperber, 2009, Turci \&
Bernarde, 2009, Sousa \& Miranda, 2010, Sobanski et al., 2012), corroborando com os resultados encontrados no presente estudo. Esta parece ser uma realidade local, uma vez que taxa semelhante foi obtida em outro trecho da mesma rodovia por Sousa \& Miranda (2010). Este fato pode ser explicado pelos cachorros-do-mato serem predadores e carniceiros e se moverem intensamente ao longo de estradas, procurando presas ou outros animais mortos por atropelamento. Além disto, trata-se de um animal que apresenta grande agilidade e uma ampla área de vida (Beisiegel, 1999, Sousa \& Miranda, 2010), possibilitando seu deslocamento por grandes distâncias a procura de alimento. A vegetação as margens da estrada, ou as próprias faixas de rolagem, podem ser utilizadas por estes animais como trilhas artificiais para sua movimentação, aumentando assim as chances de serem atropeladas (Sousa \& Miranda, 2010).

Nem todos os estudos apontam as mesmas características, uma vez que atropelamentos de animais apresentam padrões relacionados ao tipo de vegetação, clima e o comportamento das espécies (Pinowski, 2005) irá depender também da abundância da fauna em determinada região, como os resultados do trabalho de Silva et al. (2007) o trecho da BR-425 em Rondônia, apontam a classe dos répteis como a mais atingida pelos atropelamentos, principalmente Chrironius scurrulus e cobra cipó $\mathrm{Na}$ rodovia 482 Minas Gerais, a classe mais atingida foi Mammalia, onde Didelphis marsupialis, gambá de orelhas pretas, apresentando mais da metade dos casos de atropelamento.

$\mathrm{O}$ segundo grupo mais frequentemente atropelado em todo o trecho monitorado foi o dos répteis. Os resultados mostraram que $70 \%$ dos répteis atropelados correspondem a espécies de serpentes. Por possuírem deslocamento lento e necessidade de termorregular, por serem ectotérmicos, esses animais se tornam particularmente vulneráveis à mortalidade associada a estradas (Rudolph et al., 1999). Entretanto, não se pode descartar a hipótese destes atropelamentos serem intencionais, uma vez que as pessoas em diversas regiões os consideram "animais perigosos" (Moura et al., 2010), apesar de nenhuma espécie registrada ter importância médica e oferecer sérios riscos a população humana. A BR - 230 no sentido Patos à Campina - Grande possui alguns corpos d' água a sua margem, que provavelmente abrigam algumas espécies de anfíbios, mas não duram o ano todo, 
no início do período seco eles começam a diminuir e eventualmente podem vir a secar por completo, o que leva esses anfíbios a procurar áreas úmidas e alimento. Durante essa jornada acabam se deparando com a rodovia, vindo assim a serem atropelados.

Assim como encontrado por Turci \& Bernarde (2009) um maior número de registros de animais atropelados foi observado no período de seca. Estes autores associaram este resultado ao aumento do tráfego no período de seca por causa da exportação da safra produzida neste período. Esta mesma associação também foi levantada por Pereira et al. (2006) em seu estudo na Rodovia PA-458 no Pará. Entretanto, os resultados encontrados aqui não podem ser associados a este fato, uma vez que a maior produção agrícola da região é observada durante o período de chuva (Silva et al., 2009, Menezes et al., 2010). Este resultado provavelmente deve-se ao fato de que durante a estação seca os animais locomovem-se mais devido a maior escassez de recursos (Sousa \& Miranda, 2010), 2010). A sazonalidade influencia em casos de atropelamentos, visto que houve uma variação entre os períodos seco e chuvoso. Alguns táxons como aves e répteis tiveram seu maior índice de atropelamento no período de chuva, onde se destaca maior disponibilidade de alimento e o período reprodutivo dessas espécies nessa região, aumentando seu fluxo de movimentação e consequentemente elevando as chances de atropelamentos. Embora, para aves seja difícil estabelecer um padrão em relação ao período sazonal, isso ocorre provavelmente devido a grande diversidade desse grupo e a necessidade de um maior esforço amostral, o que não ocorre com frequência segundo Bager \& Da Rosa (2011).

Os resultados mostrados neste estudo, juntamente com os apresentados por Sousa \& Miranda (2010) chamam a atenção para o número de vertebrados atropelados em um pequeno trecho da BR 230, apesar de não margear nenhuma Unidade de Conservação, verificou-se índices de atropelamento de vertebrados mais elevados que os observados próximos a áreas protegidas. Neste estudo foi verificada uma taxa de 0,087 de vertebrados atropelados por quilômetro percorrido, resultado superior ao encontrado por Turci \& Bernarde (2009) na Amazônia $(0,078)$ e por Silveira (1999) (0,045 animais/Km) em rodovias que margeiam o Parque Nacional das Emas, Unidade de Conservação de 130.000 ha. Apesar de Sousa \& Miranda (2010) utilizarem outro índice para estimar o nível de atropelamento em outro trecho da rodovia utilizada no presente estudo (BR-230), também encontrou uma alta taxa de atropelamento $(0,036 /$ animais/Km/ano), fato que evidencia o impacto desta rodovia para a fauna de vertebrados.

Este fato mostra que medidas mitigadoras precisam ser executadas, como a implantação de túneis e galerias de passagem de fauna em alguns pontos da rodovia. Da mesma forma, como placas sinalizadoras, uma vez que em todo o trajeto monitorado não foi vista nenhuma sinalização que chame a atenção dos motoristas para o risco de animais na estrada. É sugerido ainda que o monitoramento dos atropelamentos deva ser realizado por um período mais amplo, além de avaliar se diferenças na vegetação junto à estrada interferem na frequência de atropelamentos.

\section{Referências Bibliográficas}

Ayres, M. 2007. BioEstat 5.0: aplicações estatísticas nas áreas das ciências biológicas e médicas. Sociedade Civil Mamirauá.

Bager, A. \& Da Rosa, C. A. 2011. Influence of sampling effort on the estimated richness of road-killed vertebrate wildlife. Environmental Management, 47, 851-858.

Beisiegel, B. M. 1999. Contribuição ao estudo da história natural do cachorro do mato, Cerdocyon thous, e do cachorro vinagre, Speothos venaticus. Universidade de São Paulo, São Paulo.

Cherem, J. J., Kammers, M., Ghizoni Júnior, I. R. \& Martins, A. 2007. Mamíferos de médio e grande porte atropelados em rodovias do Estado de Santa Catarina, sul do Brasil. Biotemas, 20, 81-96.

DNIT, 2011. Departamento Nacional de Infraestrutura de Transporte. Nomenclatura das rodovias federais. Disponível na World Wide Web em: http://www.dnit.gov.br/rodovias/rodoviasfederais/nomeclatura-das-rodovias federais/rodovias-transversais.pdf. Acesso: janeiro, 2012.

Gumier, F. C. \& Sperber, C. F. 2009. Atropelamentos de vertebrados na Floresta Nacional de Carajás, Pará, Brasil. Acta Amazonica, 39, 459-466.

Lima, S. F. \& Obara, A. T. 2004. Levantamento de animais atropelados na BR-277 às margens do Parque Nacional do Iguaçu: subsídios ao 
Programa multidisciplinar de proteção a fauna. Semana de Artes, 7, 1-6.

Menezes, H. E. A., Brito, J. I. B. \& Lima, R. A. F. A. 2010. Veranico e a produção agrícola no Estado da Paraíba, Brasil. Revista Brasileira de Engenharia Agricola e Ambiental-Agriambi, 14, 181-186.

Moreira, E. R. F. 1989. Mesorregiões e microrregiões da Paraíba: delimitação e caracterização. Gabinete do Planejamento e Acao Governamental, João Pessoa.

Moura, M. R., Costa, H. C., São-Pedro, V. A., Fernandes, V. D. \& Feio, R. N. 2010. The relationship between people and snakes in eastern Minas Gerais, southeastern Brazil. Biota Neotropica, 10, 133-141.

Pereira, A. P. F. G., Andrade, F. A. G. \& Fernandes, M. E. B. 2006. Dois anos de monitoramento dos atropelamentos de mamíferos na rodovia PA-458, Bragança, Pará. Boletim do Museu Paraense Emílio Goeldi Ciências Naturais, 1, 77-83.

Pinowski, J. 2005. Roadkills of vertebrates in Venezuela. Revista Brasileira de Zoologia, 22, 191-196.

Rudolph, D. C., Burgdorf, S. J., Conner, R. N. \& Schaefer, R. R. 1999. Preliminary evaluation of the impact of roads and associated vehicular traffic on snake populations in eastern Texas. In: Evink, G. L., Garrett, P. \& Zeigler, D. (eds.) Proceedings of the Third International Conference on Wildlife Ecology and Transportation. Tallahassee, USA.

Scoss, L. M., Marco Júnior, P., Silva, E. \& Martins, S. V. 2004. Uso de parcelas de areia para o monitoramento de impacto de estradas sobre a riqueza de espécies de mamíferos. Revista Árvore, 28, 121-127.

Silva, L. L., Costa, R. F., Campos, J. H. B. C. \& Dantas, R. T. 2009. Influência das precipitações na produtividade agrícola no Estado da Paraíba Influence of precipitations on agricultural productivity in Paraíba State. Revista Brasileira de Engenharia Agrícola e Ambiental-Agriambi, 13, 454-461.
Silva, M. O., Oliveira, I. S., Cardoso, M. W. \& Graf, V. 2007. Impacto dos atropelamentos sobre a herpetofauna da Floresta Atlântica (PR340, Antonina, Paraná). Acta Biológica Paranaense, 36, 103-112.

Silveira, L. 1999. Ecologia e conservação dos mamíferos carnívoros do Parque Nacional das Emas, Goiás. Universidade Federal de Goiás, Goiânia, Goiás.

Sobanski, E., Sabljic, D., Alm, B., Baehr, C., Dittmann, R. W., Skopp, G. \& StrohbeckKuehner, P. 2012. A randomized, waiting listcontrolled 12-week trial of atomoxetine in adults with ADHD. Pharmacopsychiatry, 45, 100-107.

Sousa, M. A. N. \& Miranda, P. C. 2010. Mamíferos terrestres encontrados atropelados na rodovia BR-230/PB entre Campina Grande e João Pessoa. Biofar: Revista de Biologia e Farmácia, 4, 72-82.

Trombulak, S. C. \& Frissell, C. A. 2000. Review of ecological effects of roads on terrestrial and aquatic communities. Conservation Biology, $14,18-30$.

Turci, L. C. B. \& Bernarde, P. S. 2009. Vertebrados atropelados na rodovia estadual 383 em Rondônia, Brasil. Biotemas, 22, 121127.

Velloso, A. L., Sampaio, E. V. S. B. \& Pareyn, F. G. C. 2002. Ecorregioes propostas para o Bioma caatinga. Recife: Associação Plantas do Nordeste, Instituto de Conservação Ambiental. The Nature Conservancy do Brasil, 74, 1-7.

Vieira, E. M. 1996. Highway mortality of mammals in central Brazil. Ciencia e Cultura, 48, 270-272.

\section{Article History:}

Received September 2017

Accepted 10 October 2017

Available on line 16 November 2017

License information: This is an open-access article distributed under the terms of the Creative Commons Attribution License 4.0, which permits unrestricted use, distribution, and reproduction in any medium, provided the original work is properly cited. 